\title{
Pulsation patterns of sinusoids vs. critical band noise
}

\author{
H. FASTL \\ Institut für Elektroakustik der Technischen Universität, Munchen, Germany
}

\begin{abstract}
Pulsation patterns of both sinusoidal and critical band "maskers" at $f_{M}=400 \mathrm{~Hz}, 1 \mathrm{kHz}, 2 \mathrm{kHz}$, and $4 \mathrm{kHz}$ are compared. At the slope towards low frequencies, the pulsation pattern of a sinusoid is up to $30 \mathrm{~dB}$ lower than the pattern of a critical band noise; at the upper slope, only small differences $(4 \mathrm{~dB})$ are noticed. Variations in the temporal configuration of the stimulus yield a great variety of pulsation patterns for one and the same "masker." Therefore, the interpretation of pulsation patterns as a quantitative measure of the ear's frequency selectivity is still obscure. On the other hand, a comparison of pulsation patterns of different "maskers," measured with one and the same stimulus paradigm, seems to be useful.
\end{abstract}

Since the classic paper of Wegel and Lane (1924), masking patterns are regarded as a measure of the frequency selectivity of the ear. For sinusoidal maskers, however, roughness and beats render the determination of the simultaneous threshold difficult; therefore, critical band maskers are frequently preferred (cf. Zwicker \& Feldtkeller, 1967). When using a forward-masking paradigm, interactions of masking tone and test tone can be minimized (e.g., Fast1, 1974a; Plomp, 1964).

A more recent method to investigate the frequency resolution of the ear is the pulsation method. In pulsation experiments, a test-tone impulse is presented alternately with a "masker" impulse. The criterion for the pulsation threshold is that an interrupted test tone sounds as if it were continuous (cf. Houtgast, 1971). This way, pulsation patterns of sinusoids (Houtgast, 1973; Warren, Obusek, \& Ackroff, 1972) which resemble masking patterns were produced. In order to point out that the criterion for pulsation threshold is the continuity of the test tone and not its masking, we refer to the stimulus component with fixed (center) frequency and SPL as "masker" in quotation marks. Since the pulsation threshold is not affected by beats, patterns of both sinusoids and critical band noise can be determined without complications (Fastl, 1974a, b; Houtgast, 1974; Warren et al., 1972).

The particular questions treated in the present paper are: (1) Are there different pulsation patterns for sinusoidal vs. critical band "maskers"? (2) Is the shape of pulsation patterns influenced by variations in the temporal configuration of the stimulus?

The author is indebted to Dipl.-Ing. H. Baumgartner for executing most of the experiments. Stimulating discussions with Prof. Dr.-Ing. E. Zwicker and Dr.-Ing. habil. E. Terhardt are gratefully acknowledged. This work was supported by Deutsche Forschungsgemeinschaft Sonderforschungsbereich "Kybernetik."

\section{METHOD AND PROCEDURE}

The experiments were performed by eight observers with normal thresholds of hearing. The sounds were presented monaurally through an earphone (Beyer DT 48S) with a free-field correction network (Zwicker \& Feldtkeller, 1967, p. 40). All thresholds were determined by the method of Békésy tracking. Each data point was measured twice in different sequences with the same fit of the earphone. Figure 1 shows the temporal pattern of the presentation.

The "masker" impulses are characterized by their sound pressure level, $\mathrm{L}_{\mathbf{M}}$. their (center) frequency, $\mathrm{f}_{\mathbf{M}}$, their bandwidth, $\Delta f_{M}$, and their duration, $T_{M}$. Likewise, the test-tone impulses are given by their SPL, $L_{T}$, test-tone frequency, $f_{T}$, bandwidth, $\Delta f_{T}$ (short-term spectrum), and duration, $\mathrm{T}_{\mathrm{T}}$. The sound pressure levels, $\mathrm{L}_{\mathrm{M}}$ and $\mathrm{L}_{\mathrm{T}}$ [re $\left.2 \cdot 10^{-5}\left(\mathrm{~N} / \mathrm{m}^{2}\right)\right]$, are measured for continuous sounds, out of which impulses with durations $T_{M}$ and $T_{T}$ are cut out. The duration $t_{g}$ of the gap between "masker" impulse and test-tone impulse is determined $20 \mathrm{~dB}$ down from the maximum of the impulses (double arrow). To minimize switching artifacts, smooth gating signals were used, as shown in Figure 1 .

The task of the observer was as follows: For a configuration such as that depicted in Figure 1, the observer heard an alternation of two sound bursts. He had to lower the level $L_{T}$ of the test-tone impulses until the tone burst sounded as if it were a continuous tone (the "masker" was still perceived as pulsating, of course). Then the observer had to raise the level $\mathrm{L}_{\mathrm{T}}$ until the tone again took on a pulsating character, and so on. In this way, the level $\mathrm{L}_{T}$ at the pulsation threshold could be read out from the Bekésy audiometer as an average of the tracking curve.

\section{RESULTS}

Figure 2 shows pulsation patterns of a sinusoid and a critical band noise, both at $f_{M}=1 \mathrm{kHz}$. The level $\mathrm{LT}_{\mathrm{T}}$ of the test tone at pulsation threshold is given as a function of test-tone frequency, $\mathrm{f}_{\mathrm{T}}$, as well as critical

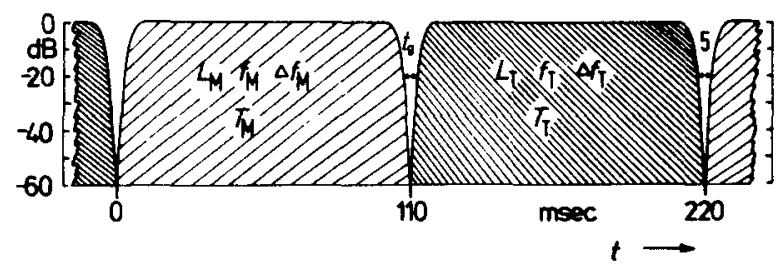

Figure 1. Stimulus paradigm. 


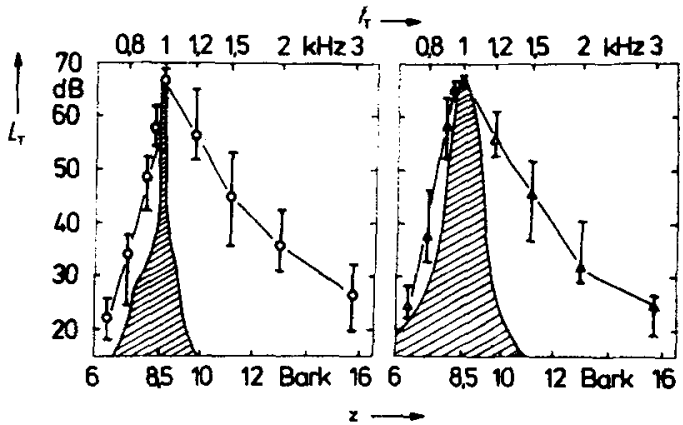

Figure 2. Palsation pattems of sinusoid and critical band notse at $1 \mathrm{kHz} . \mathrm{L}_{\mathbf{M}}=70 \mathrm{~dB}$; hatched area: spectral distribution of "masker."

band rate, $z$. The SPL of the "masker" was $\mathrm{L}_{\mathrm{M}}=$ $70 \mathrm{~dB}$. The hatched areas represent the spectral distribution of the "masker" as measured by a spectrum analyzer (Hewlett-Packard 3580A) with a resolution bandwidth of $3 \mathrm{~Hz}$. The pulsation thresholds (circles and triangles) are given as medians with interquartile ranges, each derived from 16 thresholds of eight observers.

As can be seen in Figure 2, the pulsation patterns of sinusoid and critical band noise, respectively, look quite similar.

In another series of experiments, pulsation patterns were determined for sinusoids as well as critical band noises at $f_{M}=400 \mathrm{~Hz}, 2 \mathrm{kHz}$, and $4 \mathrm{kHz}$. In each experiment, the SPL of the "masker" was $\mathrm{L}_{\mathrm{M}}=$ $70 \mathrm{~dB}$. Results are plotted in Figure 3. The filled circles and dashed lines correspond to sinusoidal "maskers," the triangles and solid lines to critical band "maskers" (cf. Zwicker, 1961). The squares represent a kind of threshold in quiet, i.e., in this case the "masker" was attenuated by $80 \mathrm{~dB}$ and the audibility of $110-\mathrm{msec}$ test-tone bursts was measured (cf. Figure 1). Each symbol in Figure 3 represents the median of 16 thresholds measured by eight observers.

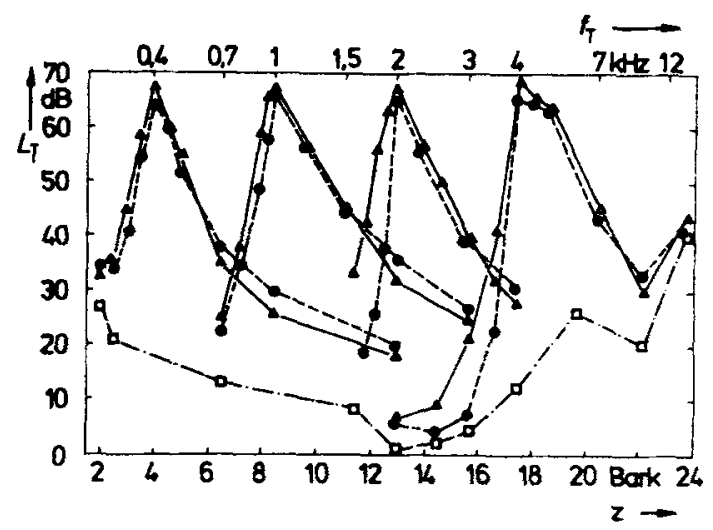

Figure 3. Pubation patterns of sinusoldal and critical band "masker." $L_{M}=70 \mathrm{~dB} ; f_{M}=400 \mathrm{~Hz}, 1 \mathrm{kHz}, 2 \mathrm{kHz}, 4 \mathrm{kHz}$; circles: sinusoldal "masker"; triangles: critical band "masker"; squares: absolute threshold.
For the sake of clarity, no interquartile ranges are given since the interquartiles depicted in Figure 2 can be regarded as typical.

The results depicted in Figure 3 suggest that sinusoids and critical band noises give rise to the same type of pulsation pattern. However, especially for $\mathbf{f}_{\mathbf{M}}$ $=2$ and $4 \mathrm{kHz}$, the pulsation pattern of the sinusoid (circles) lies below the pulsation pattern of the critical band noise (triangles). In particular, for frequencies $\mathrm{f}_{\mathrm{T}}<\mathrm{f}_{\mathrm{M}}$, differences between pulsation thresholds of sinusoidal vs. critical band "maskers" of more than $20 \mathrm{~dB}$ can be noticed. On the other hand, for $\mathrm{f}_{\mathrm{T}}>\mathrm{f}_{\mathrm{M}}$, only small differences up to $4 \mathrm{~dB}$ occur, which are of the order of magnitude of the accuracy of the measurements. The influence of the threshold in quiet (squares) on the pulsation pattern is clearly visible for $f_{M}=4 \mathrm{kHz}$.

Pulsation patterns for sinusoidal "masker" shown in Figure 3 (circles) can be compared with results of Houtgast (1973, Figure 2). While the type of pattern is essentially the same, the patterns described by Houtgast are somewhat narrower and exhibit steeper slopes. Some pilot experiments indicated that this discrepancy in the results seems to be caused by the temporal overlap of "masker" and test impulse in Houtgast's stimulus paradigm. However, the observers reported that, especially for frequencies $f_{T}$ near $\mathrm{fM}$, the determination of the pulsation threshold was complicated by interactions of "masker" tone and test tone. This means that, with an overlapping stimulus configuration, the great advantage of the pulsation method-i.e., avoiding physical interaction of "masker" tone and test tone-is lost. The shape of pulsation patterns seems to depend considerably on details of the pulsation threshold technique. A stimulus paradigm without temporal overlap of "masker" and test impulse is preferable, since for pulsation threshold "the essential condition is non-simultaneity of masker and probe-tone" (Houtgast, 1973, p. 169).

For sinusoidal "masker," Kronberg, Mellert, and Schreiner (1974) and Verschuure (1974) reported an influence of the impulse durations $\mathrm{T}_{\mathrm{M}}$ and $\mathrm{T}_{\mathrm{T}}$ on the resulting pulsation pattern. Therefore, we checked, in another experiment, whether or not the pulsation pattern of a critical band noise at $2 \mathrm{kHz}$ depends on the durations $T_{M}$ and $T_{T}$. In Figure 4, the results of two experienced observers are given as medians of eight thresholds, respectively. The SPL of the critical band noise was $\mathrm{LM}_{\mathrm{M}}=70 \mathrm{~dB}$, the durations $\mathrm{TM}=$ $\mathrm{T}_{\mathrm{T}}=30 \mathrm{msec}$ (triangles), $110 \mathrm{msec}$ (open circles), and $300 \mathrm{msec}$ (squares) were chosen. The duration of the gaps between the impulses was $t_{g}=5$ msec.

The results depicted in Figure 4 show that at the slope towards low frequencies, the pulsation threshold increases (up to $20 \mathrm{~dB}$ ) with decreasing impulse duration. The upper slope of the pulsation pattern, however, exhibits only a smaller influence of the 


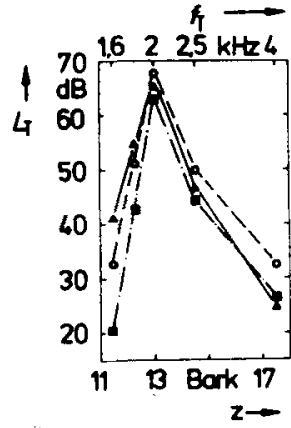

Figure 4. Pulsation patterns of critical band noise at $2 \mathrm{kHz}$. $\mathrm{L}_{\mathbf{M}}$ $=70 \mathrm{~dB} ; \mathrm{t}_{\mathrm{g}}=5 \mathrm{msec} ; \mathrm{T}_{\mathbf{M}}=\mathrm{T}_{\mathbf{T}}=30 \mathrm{msec}$ (triangles), $=$ 110 msec (open circles), $=300$ msec (squares).

impulse duration (up to $8 \mathrm{~dB}$ ). For the sinusoidal "masker," a similar behavior of the pulsation pattern was described by Verschuure (1974).

\section{DISCUSSION}

Sinusoids and critical band noises give rise to the same type of pulsation pattern. Especially at the low-frequency side. the pattern for sinusoidal "masker" is (up to $30 \mathrm{~dB}$ ) lower than the pattern for critical band "masker" (cf. Figure 3). From Figure 2, it is clear that the spectral distribution of the "masker" may influence the resulting pulsation pattern.

A comparable difference between masking patterns of sinusoid vs. critical band noise was described by Maiwald (1966, Figure 4). However, because of beats and difference tones, the masking pattern of the sinusoidal masker could only be measured for test frequencies sufficiently apart from the masker frequency.

Thus, it is an obvious advantage of the pulsation method that it yields neat patterns for both critical band and sinusoidal "maskers." On the other hand, the interpretation of pulsation patterns as a quantitative measure of the ear's frequency selectivity seems to be rather difficult:

When varying the stimulus paradigm (e.g., $T_{M}$, $\mathrm{T}_{\mathrm{T}}, \mathrm{tg}_{\mathrm{g}}$ in Figure 1), a great variety of pulsation patterns for one and the same "masker" can be found!

Shortening $T_{M}$ results in a spectral broadening of the "masker," which led to the following assumption (Verschuure, 1974): "The comparison between the spectra and the measured (pulsation) curves shows the spectrum influence to appear at the very steep low frequency side." Moreover, the duration $t_{g}$ of the gaps between "masker" and test impulse seems to influence the shape of the pulsation patterns distinctly. Obviously, the pulsation technique is considerably sensitive to alterations in the temporal stimulus pattern.

Despite these restrictions, as shown in Figure 3, a comparison of pulsation patterns of different "maskers," measured with one and the same stimulus paradigm, seems to be useful. Whereas almost complete masking patterns of sinusoids and noise bands can be measured only at very high frequencies (Fastl, 1975), pulsation patterns of both "maskers" can be compared at low as well as high frequencies.

\section{REFERENĊES}

Fastl, H. Transient masking pattern of narrow band maskers In: Facts and models in hearing. Berlin/Heidelberg/ New York: Springer, 1974. Pp. 251-257. (a)

FAstl, H. Comments on: The slopes of masking patterns. In: Facts and models in hearing. Berlin/Heidelberg/New York: Springer, 1974. Pp. 273-274. (b)

FAsTL, H. Masking patterns of subcritical vs critical band maskers at $8.5 \mathrm{~Hz}$. Acustica, 1975, 33, in press.

Houtgast, T. Psychophysical evidence for lateral inhibition. Proceedings of the 7 th International Congress on Acoustics. Budapest, 1971, 24H15.

Houtgast, T. Psychophysical experiments on "tuning curves" and "two tone inhibition." Acustica, 1973, 29, 158-179.

Houtgast. T. The slopes of masking patterns. In: Facts and models in hearing. Berlin/Heidelberg/New York: Springer. 1974. Pp. 269-272.

Kronberg, H., V. Mellert, \& Schreiner, C. Dichotic and monaural pulsation thresholds. Proceedings of the 8th Inter national Congress on Acoustics, London, 1974, Vol. I, 143.

Maiwald, D. Zusammenhang zwischen Mithorschwellen und Modulationsschwellen. Dissertation, TH Stuttgart. 1966.

Plomp, R. The ear as a frequency analyzer. Journal of the Acoustical Society of America, 1964, 36, 1628-1636.

VerschuURe, H. Transient phenomena in pulsation threshold measurements. Audiology, 1974, 13, 90 .

W ARren, R.. Orusek, C., \& ACkroff, J. Auditory induction: Perceptual synthesis of absent sounds. Science, 1972, 176. 1149-1151.

WEGEL, R. L., \& LANe, C. E. The auditory masking of one pure tone by another and its probable relation to the dynamics of the inner ear. Physics Review, 1924, 23, 266.

ZwICKER, E. Subdivision of the audible frequency range into critical bands (Frequenzgruppen). Joumal of the Acoustical Society of America, 1961, 33, 248.

Zwicker, E., \& Feldtkeller, R. Das Ohr als Nachrichtenempfänger (2. erw. Aufl.). Stuttgart: Hirzel-Verlag, 1967.

(Received for publication January 2, 1975; revision received April 27, 1975.) 